\title{
Characterization and closed-loop performance of a liquid mirror adaptive optical system
}

\author{
Eric S. ten Have ${ }^{1, *}$ and Gleb Vdovin ${ }^{1,2}$ \\ ${ }^{1}$ Delft University of Technology, Electronic Instrumentation Laboratory, Mekelweg 4, 2628 CD Delft, the Netherlands \\ ${ }^{2}$ Flexible Optical B.V., Polakweg 10-11, 2288 GG Rijswijk, the Netherlands \\ ${ }^{*}$ Corresponding author: E.S.tenHave @ tudelft.nl
}

Received 22 December 2011; revised 9 February 2012; accepted 10 February 2012;

posted 10 February 2012 (Doc. ID 160466); published 20 April 2012

\begin{abstract}
A deformable mirror based on the principle of total internal reflection of light from an electrostatically deformed liquid-air interface was realized and used to perform closed-loop adaptive optical (AO) correction on a collimated laser beam aberrated by a rotating phase disk. Equations describing the resonant and oscillatory behavior of the liquid system were obtained and applied to the system under consideration. Characterization of the mirror included open- and closed-loop frequency responses, determination of rise times, the damping times of the liquid, and the influence of liquid surface motion in the absence of external optical aberrations. The performance of the AO system was determined for static and dynamic aberrations for various sets of system parameters. The predictions of the general expressions were compared to the results of the experimental realization and were found to be in good agreement. (C) 2012 Optical Society of America
\end{abstract}

OCIS codes: $\quad 010.1080,230.6120$.

\section{Introduction}

Liquids have been used as reflecting optical surfaces in astronomy for a long time-from rotating mercury liquid mirrors [1-3] to magnetically deformable ferromagnetic liquid mirrors [4]. Because of the dissipative nature of those systems, their shaped liquid surfaces are not stable: once the driving mechanism of the rotating mercury mirrors is stopped, the liquid level will equalize and the parabolic shape will be lost. Likewise, magnetic liquid mirrors require a constant current through the coils to keep the desired shape. Additionally, even when liquid surfaces are not shaped, they are intrinsically unstable as opposed to solid surfaces normally used in flat mirrors. In adaptive optics (AO), the flexibility of a reflecting liquid surface promises to be a very useful tool when applied as a deformable mirror. Contrariwise however, the lack of rigidity may cause additional problems due

$1559-128 \mathrm{X} / 12 / 122155-09 \$ 15.00 / 0$

(C) 2012 Optical Society of America to unwanted dynamics that may introduce larger errors to an optical system than the deformable mirror corrects in the first place.

In our system, the light is reflected by total internal reflection (TIR) where the light propagates through the liquid and is reflected by an electrostatically deformed liquid-air interface. This effect is not dissipative, and the liquid mirror needs no constant source of energy to retain the shape of the surface once it is set; the electrodes act as a capacitor that will keep the field shaping the liquid surface, even if the source to the electrodes is cut off. This allows for scaling this principle to be applied in very large scale AO applications, for example, giant optical telescopes. Additionally, the use of this type of system is economically advantageous, since it is comparatively cheap to manufacture and the liquid offers a high-quality optical surface at no additional cost.

In previous work the differential equation governing the static behavior of the liquid deformation was numerically solved and stable closed-loop operation of the mirror was demonstrated [5]. Here, the theory 
describing the oscillatory behavior and possible resonances of the liquid in a container is applied to our system and compared to the measured dynamic behavior of the liquid mirror. We determine the characteristic open- and closed-loop frequency responses and investigate the dynamics of the liquid, including rise and damping times. Simultaneously, the performance of the deformable liquid mirror in an $\mathrm{AO}$ system was determined for static and dynamic aberrations for various sets of system parameters. It could be shown that the dynamics of the AO performance and the deformable liquid system are interdependent and can be correlated.

\section{Theory}

Figure 1 schematically shows the liquid mirror used for the measurements. It consists of a metal container of radius, $R$, with a transparent coupling prism mounted into the bottom of the container to allow the light to pass. The light is reflected from the surface of the liquid with a depth, $h$, which can be electrostatically deformed by applying a voltage to the electrodes positioned above the liquid surface. In our case the radius of the liquid container was about $R=$ $47.5 \mathrm{~mm}$ and the depth of the liquid was about $h=4 \mathrm{~mm}$.

The flexibility of a liquid allows us to locally shape its surface in such a way that it performs a certain function, i.e., in our case to correct a distorted wavefront. At the same time this flexibility implies that the liquid surface may be shaped by other influences outside our control. Even though the overall movement of the liquid is limited by the container, macroscopic surface motion may be excited. The shape and properties of container and liquid determine the characteristics of the allowed dynamics, i.e., the allowed normal modes of the system.

The characteristic modes form a spectrum that exhibits resonances at certain frequencies that may be excited by ambient vibrations or by the working system itself. For an application with the stated goal of reducing distortions -in this case of an optical wavefront-it is thus essential to know about the position and strength of these resonances in order to avoid interference with the regular operation of the device.

In our case the container consists of an open cylinder with smooth walls, for which the normal modes are given by

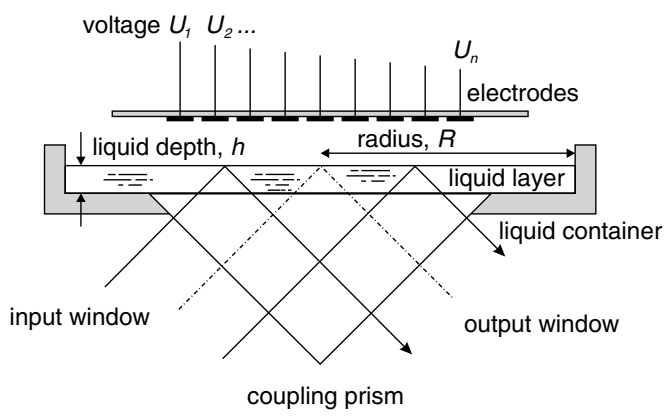

Fig. 1. Liquid mirror device.

$$
\omega_{i j}^{2}=\frac{g \xi_{i j}}{R}\left(1+\frac{\gamma \xi_{i j}^{2}}{\rho g R^{2}}\right) \tanh \left(\frac{\xi_{i j} h}{R}\right)
$$

with $R$ being the radius of the liquid container, $g$ the gravitational constant, $\gamma$ the surface tension, $\rho$ the density of the liquid, $h$ the fluid depth, and $\xi_{i j} / R$ the roots of the Bessel function $\partial J_{i}\left(\xi_{i j} r / R\right) /$ $\left.\partial r\right|_{r=R}=0$ [6] . The values of these roots can be found in the literature [7].

The corresponding wavelengths, $\lambda_{i j}$, of the modes with frequency $\omega_{i j}$ can be derived from the dispersion relation:

$$
\omega_{i j}^{2}=g k_{i j}+\frac{\gamma k_{i j}^{3}}{\rho}
$$

with $g$ being the gravitational constant, $k_{i j}$ the wavenumber, $\gamma$ the surface tension, and $\rho$ the density of the liquid [8]. With $\lambda_{i j}=2 \pi / k_{i j}$, this can be rewritten as

$$
\omega_{i j}^{2} \lambda_{i j}^{3}-2 \pi g \lambda_{i j}^{2}=\frac{8 \pi^{3} \gamma}{\rho} .
$$

This is a cubic equation that can be shown to have one real root that corresponds to the wavelength, $\lambda_{i j}$, and two nonreal complex conjugate roots that will be ignored.

The wave velocity of the modes is given by

$$
c=\frac{\omega \cdot \lambda}{2 \pi}
$$

with $c$ being the wave velocity, $\omega$ the frequency, and $\lambda$ the wavelength of the respective modes.

The frequency, wavelength, and wave velocity of the first 10 modes are summarized in Table 1 .

After excitation, these modes will be damped by a variety of effects, e.g., viscous dissipation and capillary hysteresis, at the surface and the container walls. The theory and description of this cannot always be matched very well to observations $[9,10]$. Because of the complexity of the topic, certain assumptions have to be made, one of which requires the surface boundary layer, $\delta$, to be much smaller than the liquid depth, $h$ :

Table 1. Frequency, $f$, Wavelength, $\lambda$, and Wave-Velocity, $c$, of the First 10 Modes

\begin{tabular}{lccc}
\hline & & & \\
$i j$ & $\begin{array}{c}\text { Frequency } \\
f[\mathrm{~Hz}]\end{array}$ & $\begin{array}{c}\text { Wave- } \\
\text { Wavelength }\end{array}$ & $\begin{array}{c}\text { Velocity } \\
c[\mathrm{~m}]\end{array}$ \\
\hline 10 & 1.222 & 1.046 & 1.278 \\
00 & 2.539 & 0.243 & 0.617 \\
11 & 3.529 & 0.127 & 0.448 \\
01 & 4.637 & 0.075 & 0.348 \\
12 & 5.636 & 0.053 & 0.296 \\
02 & 6.714 & 0.039 & 0.262 \\
13 & 7.727 & 0.031 & 0.241 \\
03 & 8.805 & 0.026 & 0.227 \\
14 & 9.844 & 0.022 & 0.219 \\
04 & 10.944 & 0.020 & 0.213 \\
\hline
\end{tabular}




$$
\delta=\sqrt{\frac{2 v}{\omega}} \ll h
$$

with $v$ being the kinematic viscosity of the liquid and $\omega$ the frequency of the wave [10]. In our case, the boundary layer thickness for the strongest mode, $\omega_{01}$, calculates with a kinematic viscosity of $v=$ $4.957 \cdot 10^{-5} \mathrm{~m}^{2} / \mathrm{s}$ to $\delta_{01}=1.8 \mathrm{~mm}$ while the liquid depth is $h=4 \mathrm{~mm}$. This means the requirement is not met, and applicability of the theory is limited. A more detailed analysis of this topic would be beyond the scope of this article; for a detailed discussion, see $[6,10,11]$ and references therein. A simple calculation for the damping time constant, $\tau$, of an unbounded surface is given here as a first estimate:

$$
\tau=\frac{\lambda^{2}}{8 \pi^{2} v}
$$

with $\lambda$ being the wavelength and $v$ the kinematic viscosity of the liquid [12].

\section{Setup}

Figure 2 shows the setup used to measure the openloop frequency response and the time constants of the liquid mirror. The liquid mirror consists of a metal container fitted with a coupling prism to allow the light to be reflected from the liquid surface by TIR. The radius of the liquid container is about $R=$ $47.5 \mathrm{~mm}$, and the depth is about $d=10 \mathrm{~mm}$ with a liquid depth of about $h=4 \mathrm{~mm}$. The geometry of the liquid mirror results in an effective pupil size of $9 \mathrm{~mm}$. In this experiment the liquid was an aqueous solution of $80 \%$ glycerin with a density of $\rho=$ $1208.5 \mathrm{~kg} / \mathrm{m}^{3}$ and a surface tension of about $\gamma=$ $65.7 \mathrm{mN} / \mathrm{m}$. The electrode structure used to apply a voltage and generate a static electric field to control the shape of the surface is a standard OKO electrode for a 37-ch $15 \mathrm{~mm}$ micromachined membrane deformable mirror consisting of 37 hexagonally

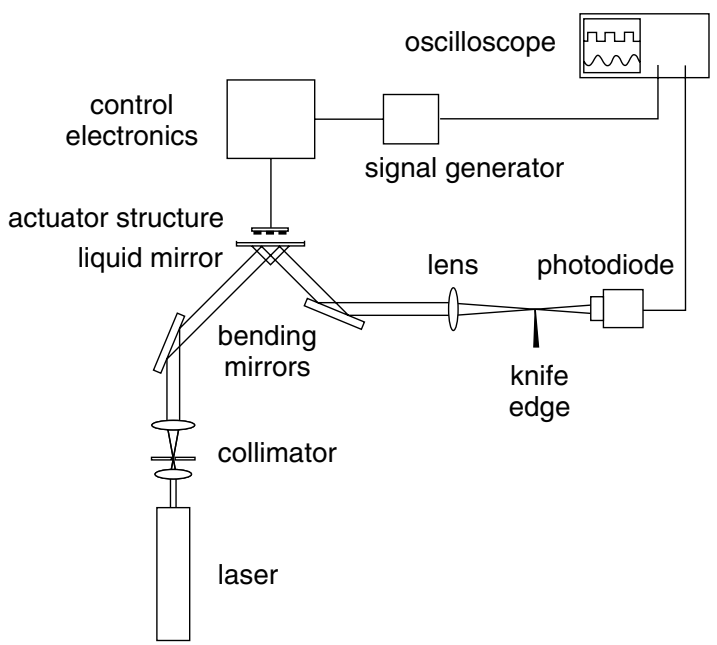

Fig. 2. Setup for the measurement of the frequency response and the time constants. arranged electrodes with a diameter of $1.9 \mathrm{~mm}$ each and an overall diameter of $15 \mathrm{~mm}$, one of which was controlled by a signal generator. The voltage applied was $U=380 \mathrm{~V}$, and the distance between the actuators and the liquid surface was about $L=700 \mu \mathrm{m}$, which resulted in a maximum stroke of about $20 \mu \mathrm{m}$.

The light reflected from the liquid mirror was focused onto a knife-edge and subsequently detected by a fast photodiode. The signals from the photodiode and the signal generator were recorded with an oscilloscope.

\section{Measurements}

\section{A. Open-Loop Characterization of the Liquid Mirror Response}

From the theory, it is expected that the frequency characteristic of the liquid mirror shows a relatively flat frequency response with peaks at the locations of the resonances predicted by Eq. (1). However, imperfections in the shape of the liquid container, i.e., irregularities in the geometry of the wall and flatness of the bottom, may cause aberrations in the spectrum. The size of these imperfections is on the order of a few $100 \mu \mathrm{m}$, leading to a broadening of the resonances of low-frequency modes (large wavelengths) and to a shift of the resonances of higher frequency modes (short wavelengths).

A Bode plot and Nichol's chart showing the openloop frequency response of the mirror are shown in Fig. 3 . The measured voltages were normalized such that the DC gain of the system equals unity, i.e., $G_{\mathrm{DC}}=1=0 \mathrm{~dB}$. While the high-frequency asymptote has a slope of about $-40 \mathrm{~dB} /$ decade and thus suggests a second-order system, there are a number of low-frequency peaks indicating an even higher order system (compare also Fig. 4). The gain crossover frequency and the phase crossover frequency of the system are given by $f_{\mathrm{gc}}=27 \mathrm{~Hz}$ and $f_{\mathrm{pc}}=115 \mathrm{~Hz}$, respectively, which leads to a gain margin of GM = $11.4 \mathrm{~dB}$ and a phase margin of $\mathrm{PM}=140^{\circ}$. Thus, the stability criteria, $f_{\mathrm{gc}}<f_{\mathrm{pc}}$ and $G<0$ at $f_{\mathrm{pc}}$, are fulfilled and the system is stable [13]. The critical delay, for which the system becomes unstable is given by $\tau_{c}=180 / f_{\mathrm{pc}}=1.57 \mathrm{~s}$.

Figure 4 shows the Fourier spectrum of the openloop liquid mirror response excited by ambient vibrations. There are two wide peaks between 2.4 and $5.4 \mathrm{~Hz}$ and around $12 \mathrm{~Hz}$, each containing multiple broadened modes and a number of narrow peaks, most notably at $19.2,27.2$, and $31.2 \mathrm{~Hz}$ that can most likely be attributed to excited modes. The corresponding measured and calculated frequencies are shown in Table 2.

The rise and relaxation times were defined as the times for the signal of the photodiode to go from $10 \%$ to $90 \%$ of its final value. The rise and relaxation times for different numbers of actuators are shown in Fig. 5(a). With the exception of the time constants for a single actuator, the development of the time 


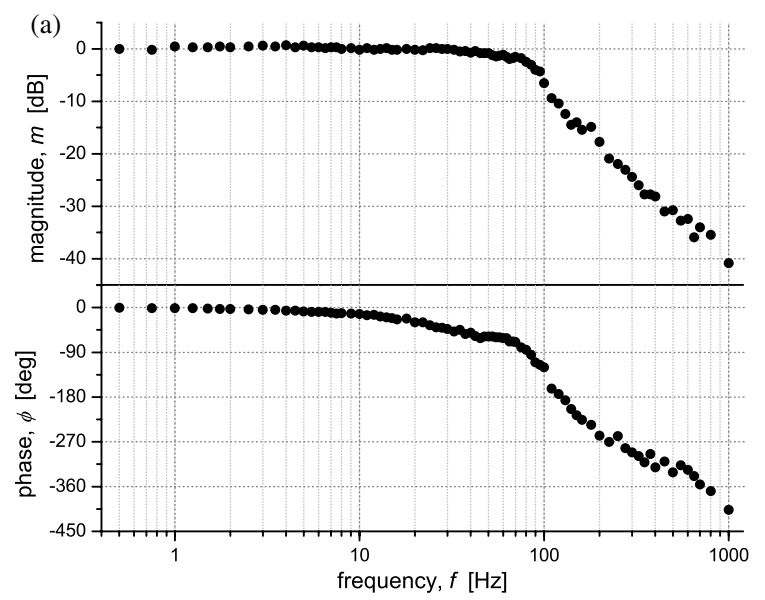

(b)

Fig. 3. Frequency response of the liquid mirror device: (a) Bode plot and (b) Nichol's chart.
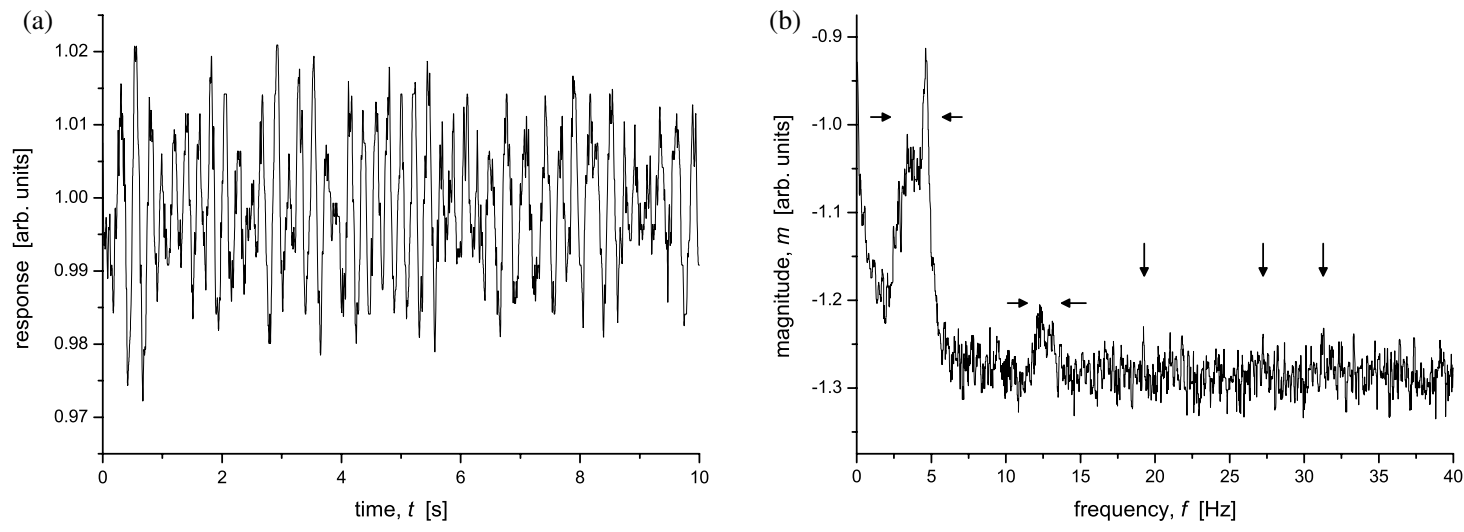

Fig. 4. Open-loop response of the liquid mirror to ambient excitations (a) in the time domain and (b) as a Fourier spectrum in the frequency domain with the frequencies of the excited modes of Table 2.

constants as a function of the number of actuators can be described by a function in the form of:

$$
\tau=a * n^{b}
$$

with $\tau$ being the rise- or relaxation-time constant of the liquid and $n$ the number of actuators with the

\footnotetext{
Table 2. Measured, $f_{\text {meas }}$, and Calculated, $f_{\text {calc }}$, Resonances of the Fourier Spectrum of the Open-Loop Liquid Mirror Frequency Response (Compare Also Fig. 12)
}

\begin{tabular}{lcc}
\hline $\begin{array}{l}\text { Measured Frequency } \\
f_{\text {meas }}[\mathrm{HZ}]\end{array}$ & $i j$ & $\begin{array}{c}\text { Calculated Frequency } \\
f_{\text {calc }}[\mathrm{Hz}]\end{array}$ \\
\hline 2.4 & & \\
& 0 & 0 \\
1 & 1 & 2.54 \\
& 01 & 3.53 \\
5.4 & & \\
\hline 11.5 & & \\
& 15 & 12.02 \\
13.4 & 05 & 13.16 \\
\hline 19.2 & & \\
27.2 & 18 & 19.13 \\
31.2 & 111 & 27.33 \\
\hline
\end{tabular}

constants $a=4.22$ and $b=1.13$ for the pulling action and $a=1.18$ and $b=1.67$ for the release action of the liquid, respectively.

The rise time as a function of the frequency is shown in Fig. 5(b). The rise time depends linearly on the frequency: first increasing to the main resonance of the system at $5 \mathrm{~Hz}$ (see Fig. 4), then decreasing again to the point that the phase lag of the system starts to increase significantly at about $15 \mathrm{~Hz}$ (see Fig. 3), and finally increasing again to the maximum rise time at about $30 \mathrm{~Hz}$, after which the accumulated phase lag prevents the signal from reaching its maximum value.

\section{B. Performance of the Closed-Loop AO System}

For the closed-loop experiments a rotating phase disk was inserted into the setup of Fig. 2 in front of the liquid mirror while the knife-edge was replaced by a wavefront sensor with 127 subapertures. The system was limited to a maximum correction frequency of about $37 \mathrm{~Hz}$ by the readout rate of the wavefront sensor camera and had an inherent system delay on the order of a few ms.

In a first step the static correction performance of the liquid mirror was measured. For different positions of the phase disk the aberrated wavefront (with 

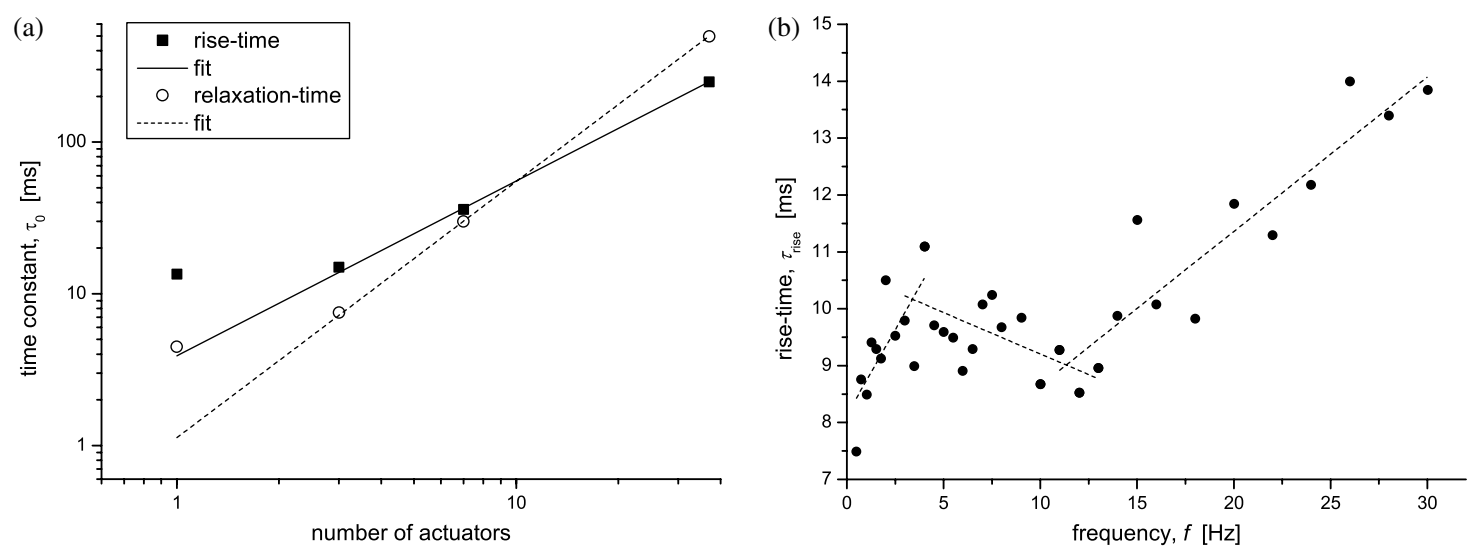

Fig. 5. Rise and relaxation time constants of the liquid mirror device as a function of (a) the number of actuators and (b) the frequency.
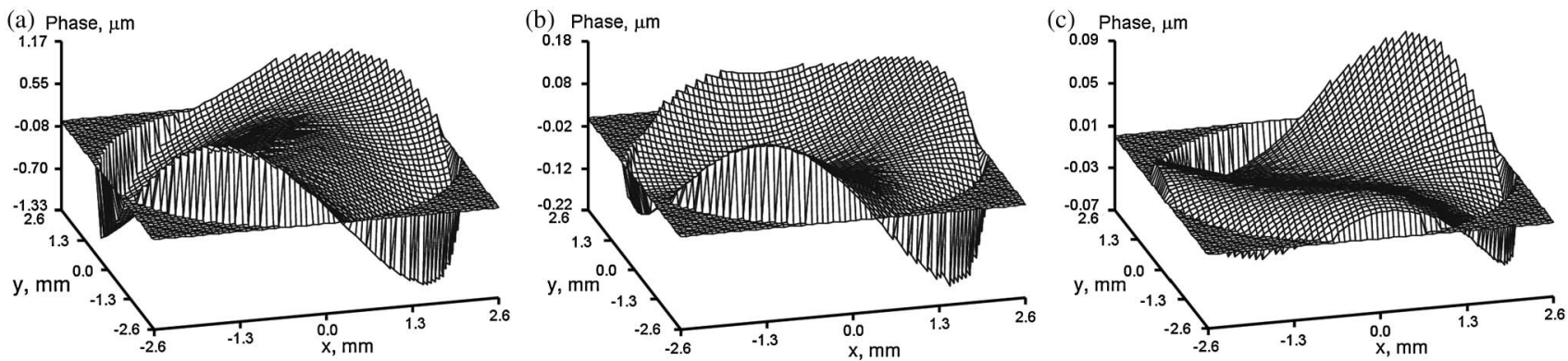

Fig. 6. Wavefronts of the (a) aberrated, (b) corrected, and (c) undisturbed beam. The image diameter is $5.2 \mathrm{~mm}$, and the peak-to-valley phase differences are $2.49,0.40$, and $0.30 \mu \mathrm{m}$, respectively.

the liquid mirror being flat) and the corresponding corrected wavefront with the feedback engaged were recorded. The correction improvement was calculated as ratio of residual wavefront rms error and Strehl ratio. Figures 6 and 7 exemplify results of the static wavefront correction: the images show the wavefronts (Fig. 6), the simulated far field (Fig. 7, top row), and the measured far field (Fig. 7, bottom row) for an aberrated (left), corrected (middle), and undisturbed beam (right). The amount of residual errors and the Strehl ratio of the beams shown are summarized in Table 3.

The average improvement, $I$, for all different positions of the phase disk was $I_{\mathrm{rms}}=6.44$ and
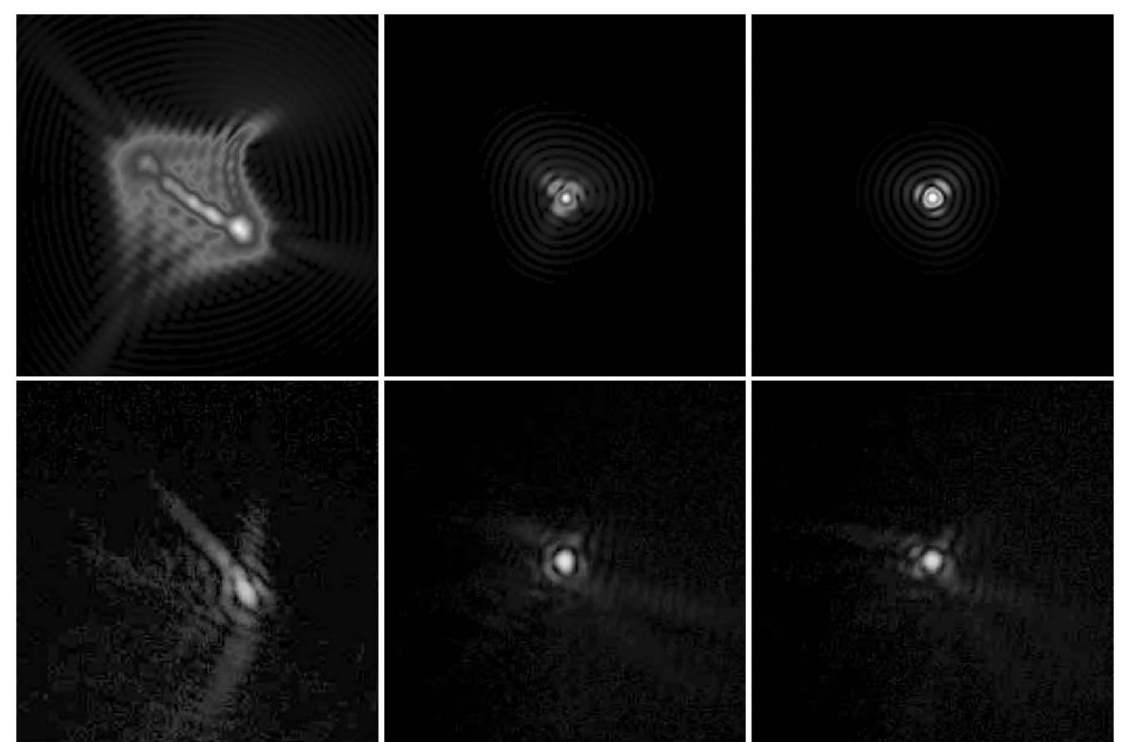

Fig. 7. Far-field images of the beam - top row: reconstruction from the wavefront sensor data, bottom row: measurement with the CCD camera behind the microscope objective. The images show the aberrated (left), corrected (middle), and undisturbed beams (right). 
Table 3. Residual Errors and Strehl Ratios of an Aberrated, Corrected, and Undisturbed Beam

\begin{tabular}{lccc}
\hline & $\begin{array}{c}\text { Aberrated } \\
\text { Beam }\end{array}$ & $\begin{array}{c}\text { Corrected } \\
\text { Beam }\end{array}$ & $\begin{array}{c}\text { Undisturbed } \\
\text { Beam }\end{array}$ \\
\hline $\begin{array}{l}\text { Peak-to-valley } \\
{[\mu \mathrm{m}]}\end{array}$ & 2.489 & 0.396 & 0.297 \\
rms $[\mu \mathrm{m}]$ & 0.421 & 0.058 & 0.042 \\
Strehl & 0.014 & 0.649 & 0.794 \\
\hline
\end{tabular}

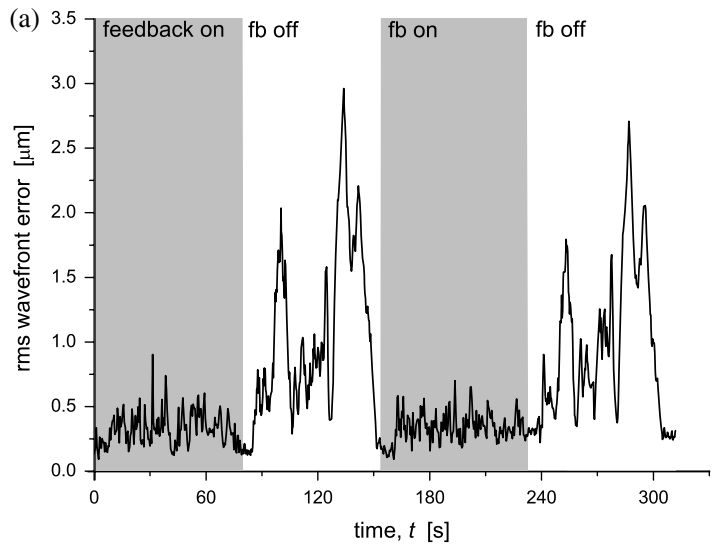

$I_{\text {Strehl }}=438$ for the ratio of the measured rms errors and Strehl ratios, respectively.

Subsequently, the dynamic correction performance of the liquid mirror was measured with the rotating phase disk for different sets of parameters. The phase disk was built from a clear plastic disk treated with hair spray and had a wedge cut out to let the beam pass undisturbed. While the phase disk was

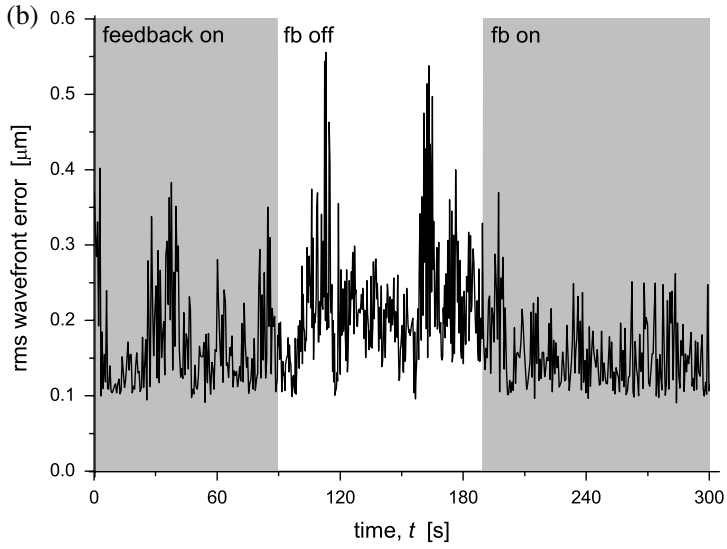

Fig. 8. Measurements of the wavefront rms error for the dynamic [(a) with the rotating phase disk] and static [(b) without phase disk] regimes; the passages with the activated feedback are shown with the gray background color. During the dynamic measurements, the feedback was switched on and off during the passage of the cut out part of the disk when the rms error was especially low.
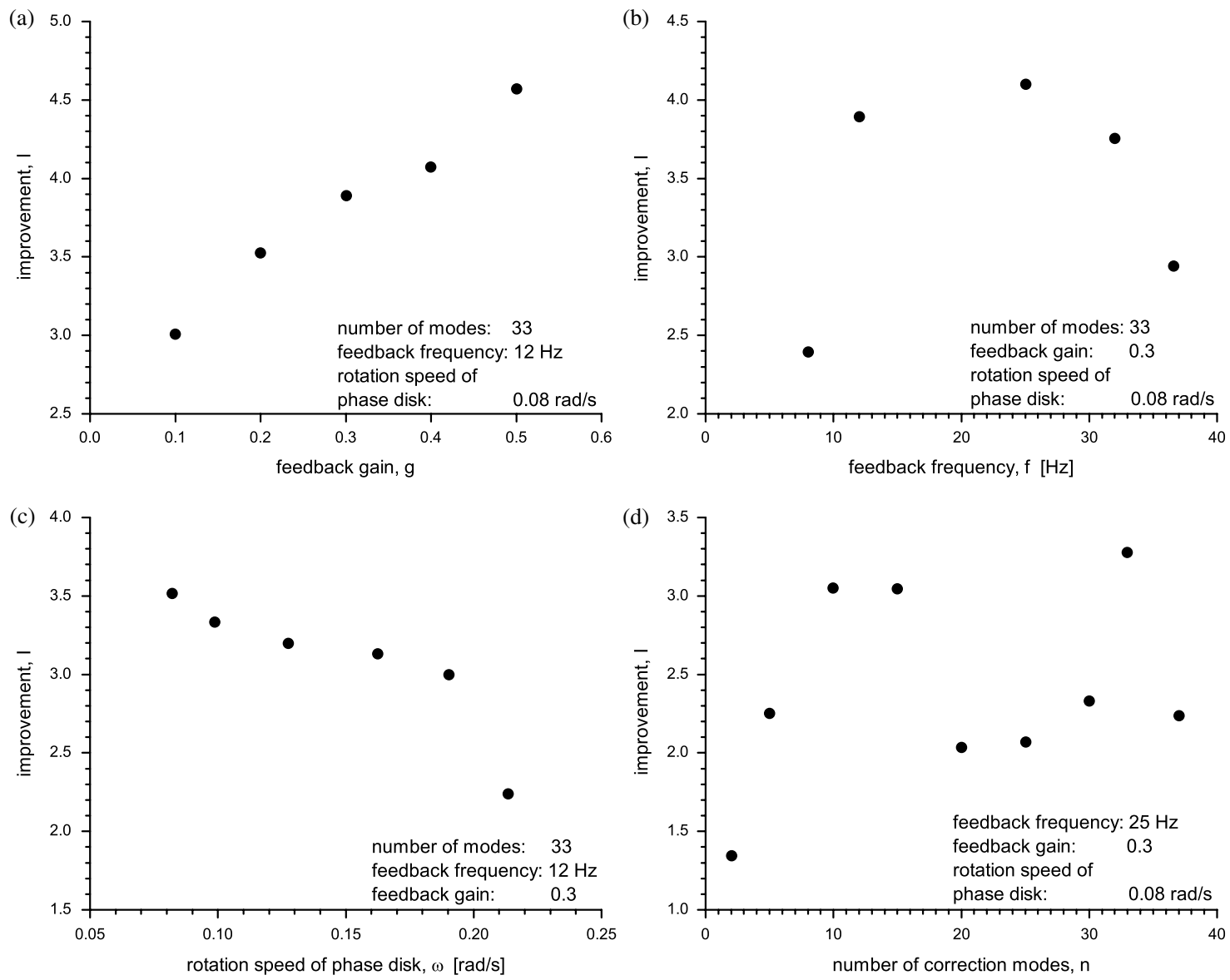

Fig. 9. Dynamic correction performance of the liquid mirror setup in terms of improvement, the ratio of the measured wavefront error induced by the rotating phase disk with and without closing the feedback loop of the liquid mirror system, as a function of (a) feedback gain, (b) feedback frequency, (c) rotation speed of the disk, and (d) number of modes. 
rotating the residual error of the wavefront was recorded with the feedback engaged and switched off [Fig. 8(a)]. The feedback was switched on or off during the passage of the cut out part of the disk so that the static aberrations of the setup were corrected for even while the feedback was switched off. A measurement of the residual error of the surface only excited by ambient vibrations is shown in Fig. 8(b) for comparison. The residual rms error of the wavefront was averaged for multiple rotations of the disk separately for active and inactive feedback and used to compute the improvement factor by dividing the average rms error without feedback by the rms error with active feedback. The rms error without feedback was relatively constant for all measurements depending only on the rotation speed of the disk, while the performance of the liquid mirror system with active feedback depended strongly on the chosen feedback parameters (Fig. 9).

Figure 9 shows the performance of the liquid mirror as a function of different system parameters: 9 (a) feedback gain, $9(\mathrm{~b})$ feedback frequency, 9 (c) rotation speed of the disk, and 9(d) number of modes.

The performance as a function of feedback frequency shows a rapid drop of the improvement factor at a low boundary frequency of about $12 \mathrm{~Hz}$ and a high boundary frequency of $32 \mathrm{~Hz}$. The lower boundary shows the effect of the maximum possible delay at which a correlation between the actual error introduced by the phase disk and the correction applied to the mirror based on the delayed error signal still exists and the influence of the resonance of the system around $12 \mathrm{~Hz}$ (compare Table 2). At the upper boundary of about $30 \mathrm{~Hz}$, the liquid mirror reaches the maximum rise time, after which the phase lag of the system prevents the liquid mirror from attaining the full amplitude of the required stroke (compare Figs. 3 and $\underline{5}$ ), so that the aberration can no longer be fully corrected.

During the closed-loop operation of the liquid mirror the error signal of the wavefront sensor was deconstructed into a superposition of Zernike modes, whose coefficients were separately recorded in real time. An example of a more detailed analysis of the first two Zernike modes (tip and tilt) is shown in Fig. 10: the polar graphs show the deflection of the beam calculated from the mode coefficients, while the bar graphs show the histograms of amplitude and angle of the deflection. By activating the feedback, the amplitude of the deflection is reduced by $73 \%$ while the variability of the angular distribution is reduced by $54 \%$.
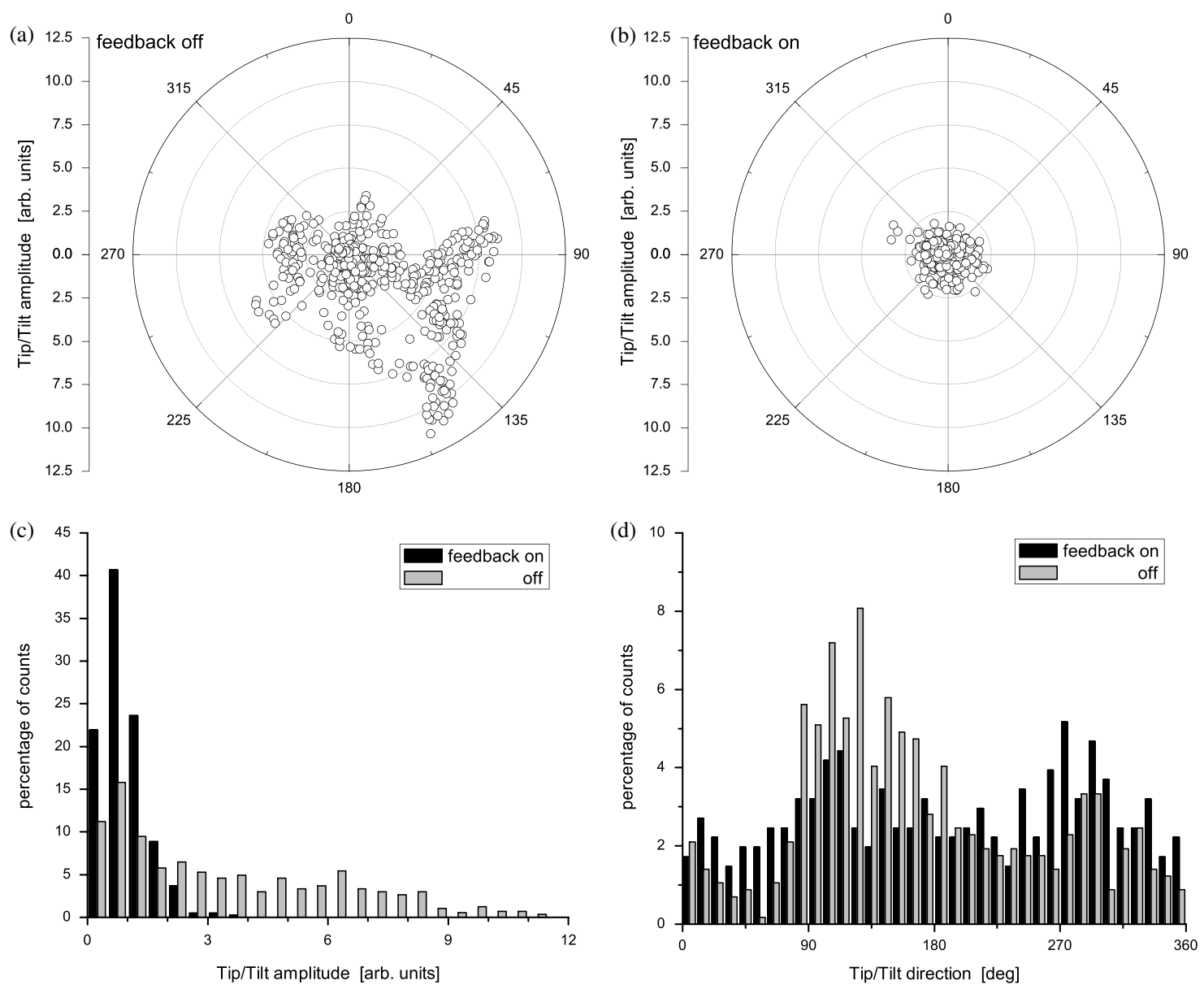

Fig. 10. Measurement of the beam deflection (a) without and (b) with closing the feedback loop. Analysis of the tip/tilt measurements shows that the liquid mirror system reduces the (c) amplitude and the (d) directional variance. 
Following the open-loop response of the system to ambient excitations (see Fig. 4), the closed-loop behavior of the liquid mirror with respect to ambient vibrations was measured [see Fig. 8(b)]. Figure 11 shows a measurement of the beam deflection by the movement of the liquid only excited by ambient vibrations without introducing wavefront errors with the phase disk.

The behavior of the system is dominated by the symmetric 01-mode (see Table $\underline{2}$ and Fig. $\underline{4}$ ), which on the basis of its symmetric geometry should not lead to a significant beam deflection for a circular beam. However, since the cross section of the beam is elongated in the direction of incidence, a measurable beam deflection along this orientation is projected onto the wavefront sensor. The polar distribution plots [Fig. 11(a) without feedback and 11(b) with feedback] show this direction to be oriented along the axis $75^{\circ}-255^{\circ}$. By closing the feedback loop the amplitude of the vibrations can be reduced by $21 \%$ [Fig. 11(c)] and the standard deviation of the angular distribution by $38 \%$. However, since the vibrations couple to the dominant resonant mode of the system the movement of the surface cannot be suppressed completely, so that the main result is the symmetrical distribution of the deflections around the origin [Fig. 11(d)].

\section{Damping}

The reduction of the excited modes through the damping of the system is also evident through comparison of the Fourier spectra of the open-loop and the closed-loop response of the system to ambient excitation: Fig. 12 shows the open-loop spectrum of Fig. 4 overlaid with the closed-loop spectrum up to $15 \mathrm{~Hz}$ together with the excited modes from Table 2 . The magnitude of the excitation is reduced over the entire spectrum and the excited modes, especially the symmetric modes $\omega_{0 i}$, are significantly damped.

The damping times for the system were measured for the free surface as $\tau_{\text {free }}=1.83 \pm 0.16 \mathrm{~s}$ and for the closed-loop system as $\tau_{\mathrm{fb}}=1.58 \pm 0.16 \mathrm{~s}$, while theory [Eq. ( 6 )] predicts $\tau_{\text {theory }}=1.44 \mathrm{~s}$. This shows that the measured times are of the right order of magnitude and that the damping time is reduced by the feedback system by about $14 \%$.
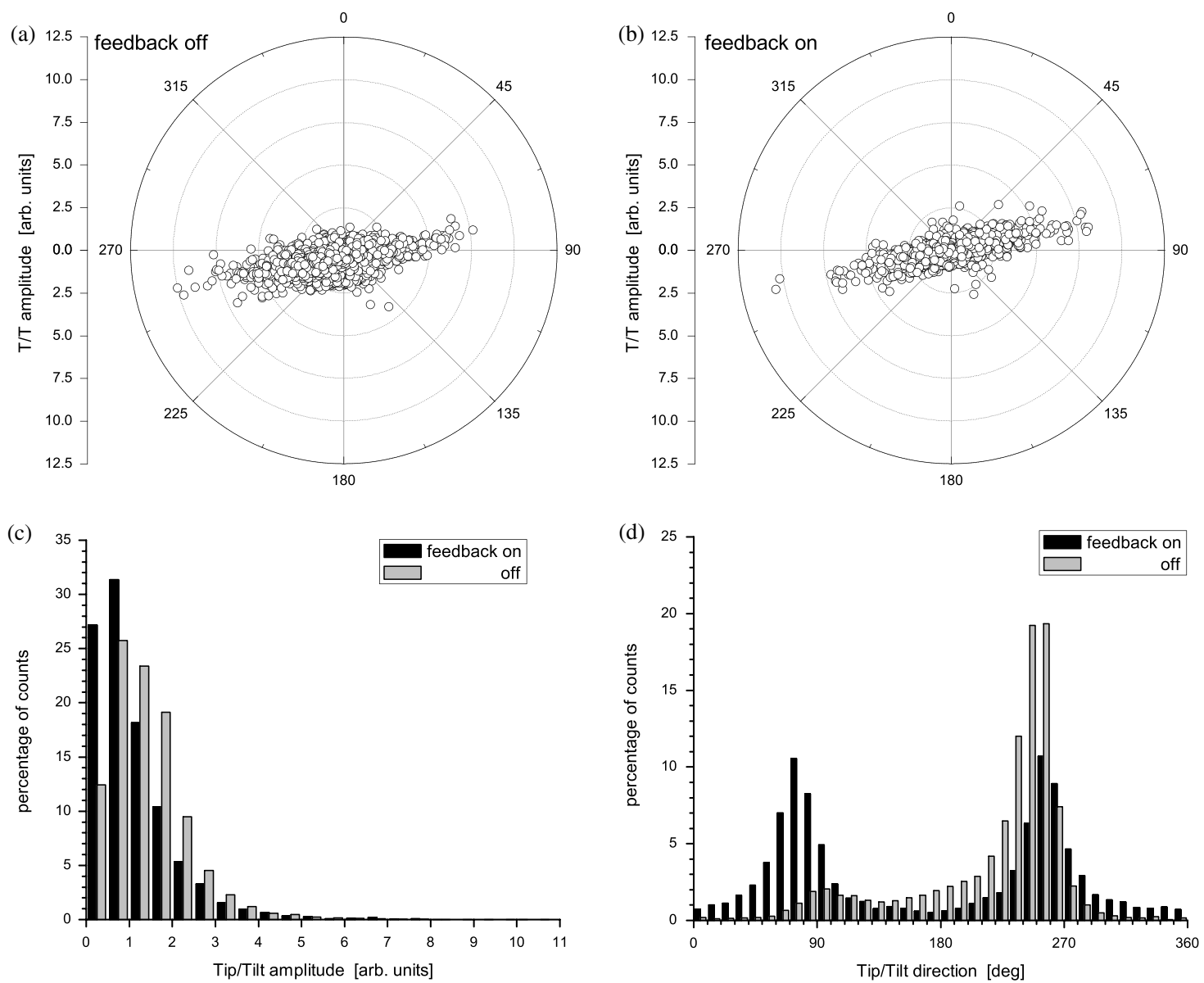

Fig. 11. Measurement of the beam deflection (a) without and (b) with closing the feedback loop. The axis of deflection oriented along $75^{\circ}-255^{\circ}$ coincides with the direction in which the cross section of the beam is elongated on the liquid mirror and thus with its direction of incidence on the liquid surface. Analysis of the tip/tilt measurements shows that the liquid mirror system (c) reduces the amplitude and (d) distributes the deflection symmetrically around the center. 


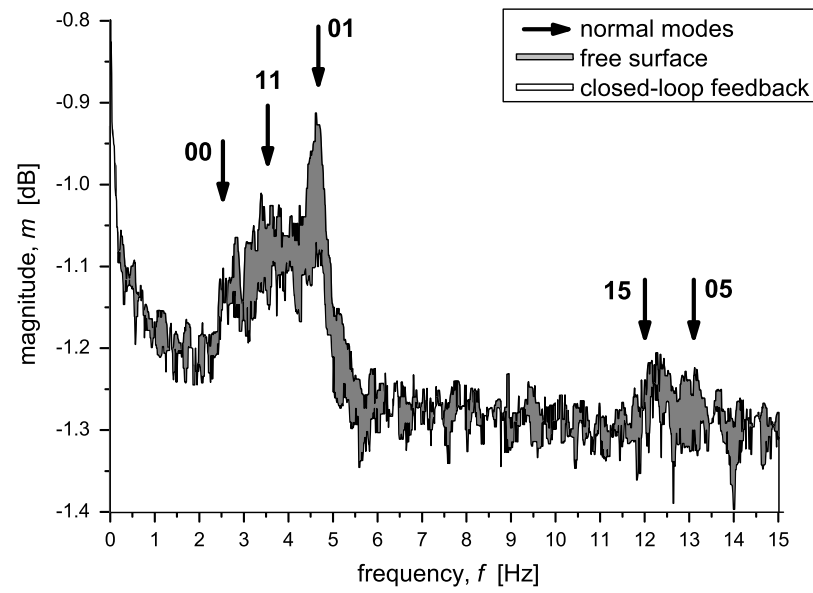

Fig. 12. Fourier spectra of the liquid mirror response with a free surface (open-loop) and with closed-loop operation of the system. The arrows show the positions of the normal modes of the liquid container (compare Table 2).

\section{Conclusions and Summary}

We have measured the open-loop frequency response and the time constants of the liquid mirror. The Bode plot and Fourier spectrum of the open-loop liquid mirror response show that the liquid mirror system is a higher order transfer function with the symmetric 01 -mode at $4.6 \mathrm{~Hz}$ as the main resonance predicted by sloshing theory. Both the gain margin, $\mathrm{GM}=11.4 \mathrm{~dB}$, and the phase margin, $\mathrm{PM}=140^{\circ}$, predict that the system is stable, thus allowing the use of the liquid mirror in a closed-loop AO system. The time constants of the liquid mirror increase with the number of actuators, $n$, since increasingly more liquid has to be displaced. Since both the frequency response and the time constants depend both on the geometry of the liquid container and the material properties of the liquid, it is possible to tune the characteristics of the system in order to optimize the performance of the mirror. The resonant behavior of the system is mainly determined by the radius of the dish and the depth of the liquid. Here, a smaller dish will increase the resonant frequencies [Eq. (1)], which will make it more difficult for the lowfrequency ambient vibrations (e.g., building vibrations) to couple to the liquid mirror. Also, a smaller liquid depth will increase the damping [Eq. (5)] and thus enhance the dissipation of the energy coupled to the liquid. The time constants and the maximum possible stroke of the system on the other hand are mainly determined by the properties of the liquid and may be tuned by choosing a liquid with an optimal combination of density, surface tension, and viscosity. However, more work on the influence of the material properties of the liquid and the geometry of the liquid container have to be done.

In accordance with the predicted stability of the system, the liquid mirror performed well in a closed-loop AO system and did improve a laser beam aberrated by a phase disk. Static and dynamic measurements were performed and showed an average improvement factor in terms of the residual rms error of $I_{\text {rms,static }}=6.44$ for the static case and of around $I_{\mathrm{rms} \text {,dynamic }}=3.5$ for the dynamic case depending on the system parameters.

Extended measurements of the tip and tilt modes of the unaberrated beam showed the beam deflection due to coupling of ambient vibrations to the normal modes of the liquid container. It could be shown that by activating the feedback the excited normal modes could be damped and the oscillations could be distributed symmetrically around the origin.

Because of the simple architecture of the system, the area and thus the number of actuators of the liquid mirror is in principle unlimited; in reality however, the size of the mirror will be limited by practical considerations. Nevertheless, the liquid mirror can be manufactured very cost effectively and can potentially be applied to very large scale AO systems, for example in future giant optical telescopes.

\section{References}

1. R. W. Wood, "The mercury paraboloid as a reflecting telescope," Astrophys. J. 29, 164-176 (1909).

2. E. F. Borra, M. Beauchemin, and R. Lalande, "Liquid mirror telescopes: observations with a 1 meter diameter prototype and scaling-up considerations," Astrophys. J. 297, 846-851 (1985).

3. P. Hickson, E. F. Borra, R. Cabanac, R. Content, B. K. Gibson, and G. A. H. Walker, "UBC/Laval 2.7 meter liquid mirror telescope," Astrophys. J. 436, L201-L204 (1994).

4. D. Brousseau, E. F. Borra, M. Rochette, and D. B. Landry, "Linearization of the response of a 91-actuator magnetic liquid deformable mirror," Opt. Express 18, 8239-8250 (2010).

5. G. Vdovin, "Closed-loop adaptive optical system with a liquid mirror," Opt. Lett. 34, 524-526 (2009).

6. R. A. Ibrahim, Liquid Sloshing Dynamics, Theory and Applications (Cambridge University, 2005).

7. H. F. Bauer, "Tables of zeros of cross product Bessel functions," J. Math. Comput. 18, 128-135 (1964).

8. L. Landau and E. Lifshitz, Fluid Mechanics, 2nd ed., Vol. 6 of Course of Theoretical Physics (Butterworth Heinemann, 2009).

9. J. Dong, J. Qi, and R. Miao, "Measurement of the damping of liquid surface wave by diffraction method," Braz. J. Phys. 37, 1129-1133 (2007).

10. J. Miles, "Surface-wave damping in closed basins," Proc. R. Soc. Lond. A 297, 459-475 (1967).

11. C. Mei and L. Liu, "The damping of surface gravity waves in a bounded liquid," J. Fluid. Mech. 59, 239-256 (1973).

12. H. Lamb, Hydrodynamics,6th ed. (Cambridge University, 1975).

13. J. J. DiStefano, A. R. Stubberud, and I. J. Williams, Outline of Theory and Problems of Feedback and Control Systems, 2nd ed., Schaum's Outline Series (McGraw-Hill, 1990). 